\title{
Particle Swarm Optimization Guided Genetic Algorithm: A Novel Hybrid Optimization Algorithm
}

\author{
V. Jagan Mohan ${ }^{\# 1}$, T. Arul Dass Albert ${ }^{\# 2}$ \\ Department of Electrical \& Electronics Engineering, \\ Karpagam Academy of Higher Education, Coimbatore, India \\ 1jaganmohansla@yahoo.co.in \\ 2 drtaruldossalbert@gmail.com
}

\begin{abstract}
In real world applications, optimization is an inevitable stage in any engineering design. In recent days the optimization theory is also fused into other sciences which require precision in its final result. This topic sounds like a promising domain for research almost in all areas of science and technology. Perhaps several solution methods are proposed for solving problems that require optimization algorithms, in that also the algorithms inspired by natural selection are dominant among them. This paper proposes a hybrid algorithm that integrates two well established methods, one the genetic algorithm (GA) and the other the particle swarm optimization (PSO) algorithm. Here the GA will be the main optimizer and the PSO will be used to guide the GA to locate optimal solutions quickly and effectively. Several benchmark test problems are solved and the applicability of the proposed hybrid algorithm is well established.
\end{abstract}

Keyword- Genetic Algorithm, Particle Swarm Optimization, Soft Computing, Benchmark Problems

\section{INTRODUCTION}

In recent days, real world optimization problems any engineering design is a real challenge. Similarly, the optimization theory is also under pining into other sciences which requires the application of cost based design. This topic emerges as a promising topic for research almost in almost diverse fields of science and technology. As said earlier, several solution methods are developed for solving optimization problems, among them the algorithms inspired by natural selection are dominant [1]. In this introduction section, a brief review of literature will be reviewed basically with an intention only on hybrid algorithms that integrates two well established methods, one the genetic algorithm (GA) and the other the particle swarm optimization (PSO) algorithm.

In [1], a new evolutionary learning algorithm is based on a hybrid of genetic algorithm (GA) and particle swarm optimization (PSO) called HGAPSO is proposed. Here individuals in a new generation are created, not only by crossover and mutation operation as in GA, but also by PSO. In another research is [2], a hybrid method combining genetic algorithms (GA) and particle swarm optimization (PSO), for the global optimization of multimodal functions is developed. [3] proposes a solution improvement phase can be assisted by knowledge stored within the parent solutions, effectively allowing parents to teach their offspring how to improve their fitness. In this paper, the evolution of each individual of the total population, which consists of the parents and the offspring, is realized with the use of a Particle Swarm Optimizer.

Subsequently, a supply chain management with bi-level linear programming to supply chain distribution problem was solved using hybrid of genetic algorithm (GA) and particle swarm optimization (PSO) [4]. In another work, [5], a hybrid Particle Swarm Optimization (PSO) and Genetic Algorithm (GA) method is used for gene selection, and Support Vector Machine (SVM) is adopted as the classifier. In [6], a novel PSO-GA-based hybrid algorithm with "dying probability" for the individuals and the "war/disease process" for the population. [7] introduces, Hybrid Particle Swarm Optimization and Genetic Algorithm (HPSOGA) is proposed to solve the multi-UAV formation reconfiguration problem, which is modeled as a parameter optimization problem.

In [8] an evolutionary-based clustering algorithm based on a hybrid of genetic algorithm (GA) and particle swarm optimization algorithm (PSOA) for order clustering in order to reduce surface mount technology (SMT) setup time. Also in [9], novel combined genetic algorithm (GA)/particle swarm optimization (PSO) is presented for optimal location and sizing of DG on distribution systems. Subsequently in [10], Adaptive Genetic Algorithm (AGA) and Particle Swarm Optimization (PSO) are implemented to get optimal schedules and storage assignments for Flexible Manufacturing Systems.

Based on the above review of literature confined only to the hybridization of genetic algorithm (GA) and the particle swarm optimization (PSO) algorithm, the functional integration proposed in this research is unique of its kind. It is demonstrated that the extensive search behaviour of GA and the exhaustive search capability of PSO are superior in searching better optimal points. In the following section we will discuss in detail the proposed functional fusion of GA and PSO. 


\section{Proposed hybrid ALgorithm}

This section will describe how two powerful optimization algorithms inspired by natural selection are integrated together to effectively solve complex optimization problems. Two powerful algorithms widely applied in several literature to solve complex engineering optimization problems: one is GA and the other is PSO. Since both the algorithms are sufficiently discussed in detail in several published literatures, this paper will give an overview of these methods and will describe the proposed hybrid GA-PSO method subsequently. it.

\section{A. Genetic Algorithm: An Overview}

GA is a randomized search method inspired based on biological evolutionary rue of survival of the fittest [11]. GA starts its search from the set of population strings that are assumed as potential solutions, randomized within the search range. Inspired by natural evolution, the algorithm will evolve new potential solutions called as offsprings from previous parents. Perhaps GA is limited for exploration features, causing slow or poor convergence and poor robustness. Thus problems like premature convergence and trapping local optima are prone when dealing complex optimization problems. it.

\section{B. Particle swarm optimization: An Overview}

PSO is one of the latest evolutionary techniques developed by Eberhart and Kennedy [12,13]. PSO is inspired based on social interaction of bird flocking or fish schooling. The particles also called as potential solutions, move throughout the multidimensional solution space and the positions of each particles were adjusted according to its own best position, and best among all its group. PSO does fall under the survival of the fittest algorithm as the entire group will be used from beginning to end. Slow or poor convergence and poor robustness are again a demerit for PSO.

\section{Proposed architecture of the hybrid GA-PSO:}

The main idea behind this proposal is, after the fitness evaluations are made for each parent in the present population, selection will be done using roulette wheel and two parents are selected based on their fitness ranking and crossover will be performed. In this crossover, two parents will share their chromosomes to produce two off-springs, which will be the new parent in the next generation.

Unlike this regular crossover, which will lead to possible dramatic change in search direction due to crossover exchange, among this two parents the best individual will be used as gbest and the other one will be retained as pbest. Thus only one parent will be disturbed and produced by this operation derived from PSO. This will help the hybrid algorithm to search the space exhaustively. Additionally this PSO operation will not be done for the entire crossover phase, instead a random of $50 \%$ in the beginning stage and gradually reduced to $5 \%$ of the total population in the entire run of the algorithm.

$$
\begin{gathered}
x_{i}^{k+1}=x_{i}^{k}+v_{i}^{k+1} \\
v_{i}^{k+1}=v_{i}^{k}+c_{1} \operatorname{rand}\left(x_{i}^{k}-p b\right)+c_{2} \operatorname{rand}\left(x_{i}^{k}-g b\right)
\end{gathered}
$$

\begin{tabular}{|c|c|c|c|c|c|c|c|c|c|c|c|c|c|}
\hline 1 & 1 & 0 & 1 & 0 & 0 & 1 & 0 & 0 & 1 & 0 & 0 & 1 & 0 \\
\hline \multicolumn{14}{|c|}{ Parent B } \\
\hline 1 & 0 & 0 & 0 & 1 & 0 & 1 & 0 & 1 & 1 & 1 & 0 & 0 & 1 \\
\hline \multicolumn{14}{|c|}{ Offspring } \\
\hline 1 & 0 & 0 & 0 & 1 & 0 & 1 & 0 & 0 & 1 & 0 & 0 & 1 & 0 \\
\hline
\end{tabular}

Parent A

Fig. 1. (a) Crossover operation in GA

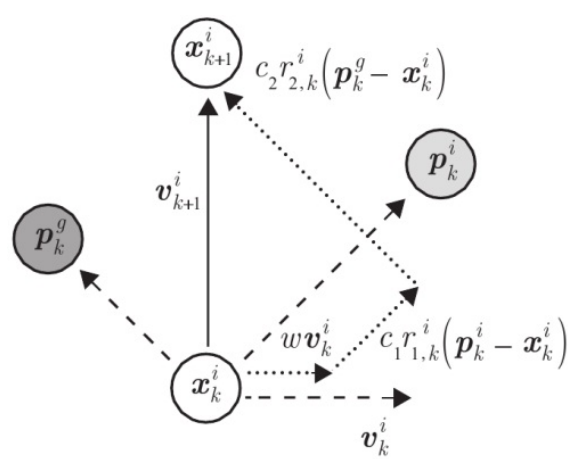

(b) Velocity and position update in PSO

As an example, let us assume two parents are selected for crossover as shown in Fig 1. The fitness value for parent A, B and offspring is 13458, 8889, 8850 respectively. Now instead of this, we use a velocity equation to update the position of parent $\mathrm{B}$ using the idea derived from PSO and the equation is given for reference.

Here the gbest is 13458 , pbest is 8889 . With regular parameter setting the new position for parent $\mathrm{B}$ will be estimated as 7452 using the velocity and position update equation in $(1 \& 2)$. Thus the possibility of arriving at better results will be large when going for this hybridization approach. Thus the proposed shift of production of new population in GA will be guided by PSO with a 50\% probability is established. A detailed flowchart of the proposed hybrid GA-PSO algorithm is shown in figure 2. 


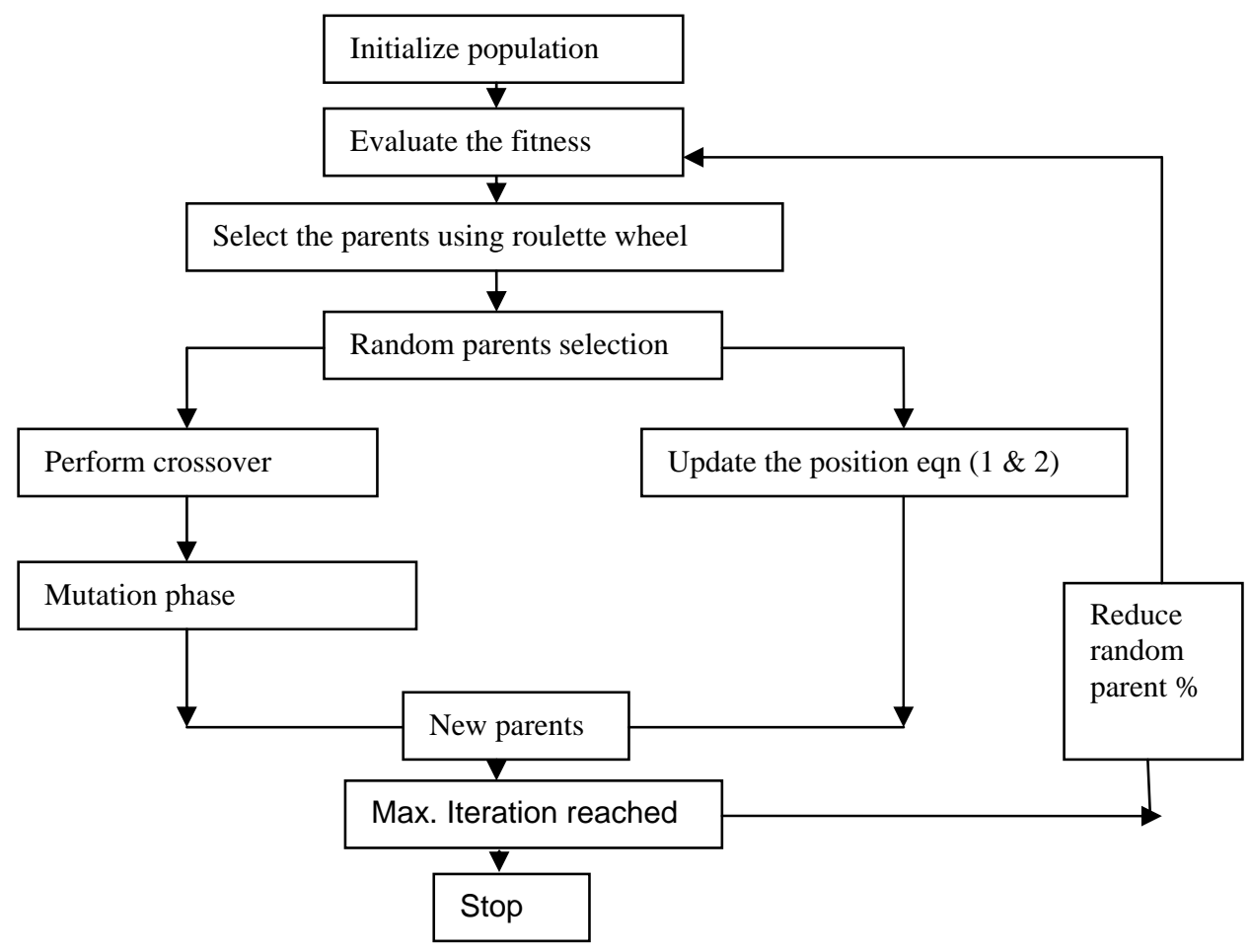

Fig. 2. Flowchart of the proposed hybrid GA-PSO

The percentage of random parents selected will be reduced during the course of run from $50 \%$ to $5 \%$ using the following equation (3):

$$
\text { rndprnt }=((0.5-0.05) / \max \text { iter }) \times \text { iter }
$$

In the next section the proposed algorithm will simulated for a sample of 10 benchmark problems widely used for simulation studies.

\section{III.NUMERICAL ILLUSTRATIONS}

In order to validate the performance of the proposed hybrid GA-PSO algorithm and demonstrate its superiority over the other algorithms in finding the better solutions, 10 standard bench mark problems are solved. These benchmark problems are widely used in several literatures [14,15]. All the simulations are carried out with following simulation parameters as summarized in Table 1.

TABLE I. Simulation parameters

\begin{tabular}{|c|c|c|c|}
\hline Method & Population & Max. Iteration & Probability / Inertia \\
\hline GA & 40 & 5000 & 0.8 and 0.001 \\
\hline PSO & 40 & 5000 & 0.9 to 0.4 \\
\hline
\end{tabular}

The proposed hybrid algorithm also uses the same parameters appropriately at the stage when the techniques are used. The objective functions, constraints and their predetermined solutions are listed in Table 2 for easy reference. The purpose of this paper is now well validated from the simulation results.

Simulation results are shown in Table 3.The experiment compares the Hybrid GA-PSO to other algorithms such as PSO and GA. The results for maximum iterations are reported in Table 3taking into consideration the following performance indexes: the Best optimum so far, the mean of the Best solution and the Standard Deviation of optimum solution obtained so far. The best result from the proposed method is summarized for each benchmark function. Observing this table, the proposed GA-PSO searches better results than GA and PSO for all tried functions. In particular, the experiment records the significant difference in demonstration which deliberately illustrates the better trade-off between exploration and exploitation in the search space. 
TABLE II. Benchmark functions

\begin{tabular}{|c|c|}
\hline \multicolumn{2}{|c|}{ Wayburn Seader 1 Function } \\
\hline \multicolumn{2}{|l|}{$f_{102}(x)=\left(x_{1}^{6}+x_{2}^{4}-17\right)^{2}+\left(2 x_{1}+x_{2}-4\right)^{2}$} \\
\hline subject to $-500 \leq 500$ & $x^{*}=f\{(1,2),(1.597,0.806)\}, f\left(x^{*}\right)=0$ \\
\hline \multicolumn{2}{|c|}{ Wayburn Seader 2Function } \\
\hline \multicolumn{2}{|c|}{$f_{163}(X)=\left[1.613-4\left(x_{1}-0.3125\right)^{2}-4\left(x_{2}-1.625\right)^{2}\right]^{2}+\left(x_{2}-1\right)^{2}$} \\
\hline subject to $-500 \leq 500$. & $X^{*}=f\{(0.2,1),(0.425,1)\}, f\left(X^{*}=0\right)$ \\
\hline \multicolumn{2}{|c|}{ Wayburn Seader 3 Function } \\
\hline \multicolumn{2}{|c|}{$f_{164}(X)=2 \frac{x_{1}^{3}}{3}-8 x_{1}^{2}+33 x_{1}-x_{1} x_{2}+5+\left[\left(x_{1}-4\right)^{2}+\left(x_{2}-5\right)^{2}-4\right]^{2}$} \\
\hline subject to $-500 \leq 500$ & $X^{*}=f(5.611,6.187), f\left(X^{*}\right)=21.35$ \\
\hline \multicolumn{2}{|c|}{ Wolfe Function } \\
\hline \multicolumn{2}{|l|}{$f_{168}(X)=\frac{4}{3}\left(x_{1}^{2}+x_{2}^{2}-x_{1} x_{2}\right)^{0} .75+x_{3}$} \\
\hline Subject to $0 \leq x_{i} \leq 2$ & $X^{*}=f(0, \cdots, 0), \quad f\left(x^{*}\right)=0$. \\
\hline \multicolumn{2}{|c|}{ Zettl Function } \\
\hline \multicolumn{2}{|l|}{$f_{174}(X)=\left(x_{1}^{2}+x_{2}^{2}-2 x_{1}\right)^{2}+0.25 x_{1}$} \\
\hline Subject to $-5 \leq x_{i} \leq 10$ & $X^{*}=f(-0.0299,0), f\left(X^{*}\right)=-0.003791$ \\
\hline \multicolumn{2}{|c|}{ Sphere Function } \\
\hline \multicolumn{2}{|l|}{$f_{137}(x)=\sum_{i=1}^{D} X_{i}^{2}$} \\
\hline Subject to $0 \leq X_{i} \leq 10$ & $x^{*}=f(0, \ldots, 0), f\left(x^{*}\right)=0$ \\
\hline \multicolumn{2}{|c|}{ Stepint Function } \\
\hline \multicolumn{2}{|l|}{$f_{141}(x)=25+\sum_{i=1}^{D}\left(x_{i}^{2}\right)$} \\
\hline Subject to $-5.12 \leq X_{i} \leq 5.12$. & $x^{*}=f(0, \ldots, 0), f\left(x^{*}\right)=0$ \\
\hline \multicolumn{2}{|c|}{ Styblinski-Tang Function } \\
\hline \multicolumn{2}{|l|}{$f_{144}(x)=\frac{1}{2} \sum_{i=1}^{n}\left(X_{i}^{4}-16 X_{i}^{2}+5 X_{i}\right)$} \\
\hline Subject to $-5 \leq X_{i} \leq 5$ & $x^{*}=f(-2.903534,-2.903534), f\left(x^{*}\right)=-78.332$ \\
\hline \multicolumn{2}{|c|}{ Trecanni Function } \\
\hline \multicolumn{2}{|l|}{$f_{149}(x)=X_{1}^{4}-4 X_{1}^{3}+4 X_{1}+X_{2}^{2}$} \\
\hline Subject to $-5 \leq X_{i} \leq 5$. & $x^{*}=f(\{0,0\},\{-2,0\}), f\left(x^{*}\right)=0$ \\
\hline \multicolumn{2}{|c|}{ Zirilli or Aluffi-Pentini's Function } \\
\hline \multicolumn{2}{|l|}{$f_{175}(X)=0.25 x_{1}^{4}-0.5 x_{1}^{2}+0.1 x_{1}+0.5 x_{2}^{2}$} \\
\hline Subject to $-10 \leq x_{i} \leq 10$ & $X^{*}=(-1.0465,0), \quad f\left(X^{*}\right) \approx-0.3523$ \\
\hline
\end{tabular}


Figure 3 presents the convergence curves for all the methods PSO, GA and the proposed algorithm considering as benchmark functions (a) F162, (b) F163, (c) F164, (d) F168, (e) F144, (f) F145, (g) F174, and 9h) F175, from the experimental set. Among them, the rate of convergence of GA-PSO is the fastest, which explores the solution space swiftly and determines the best solution in less of 500 iterations in an average compared to the other two algorithms.

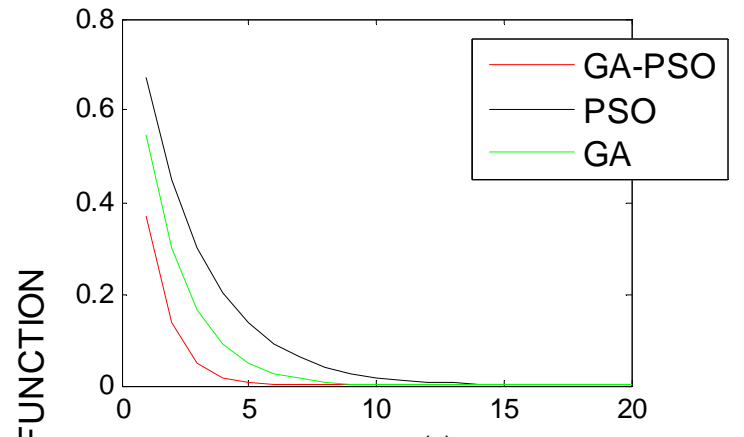

(a)

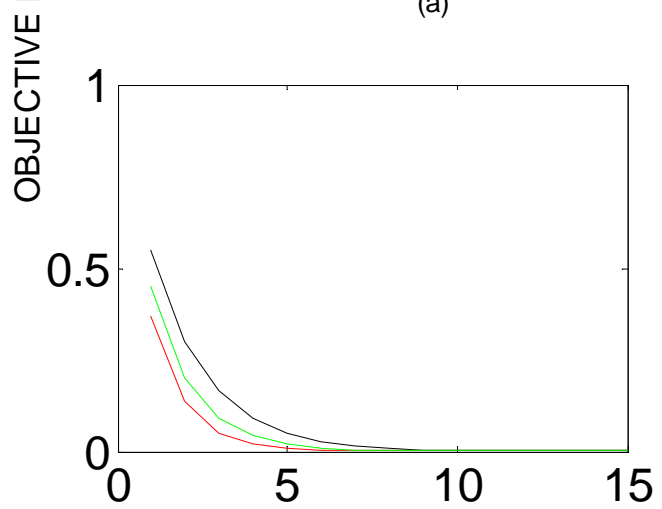

(c)

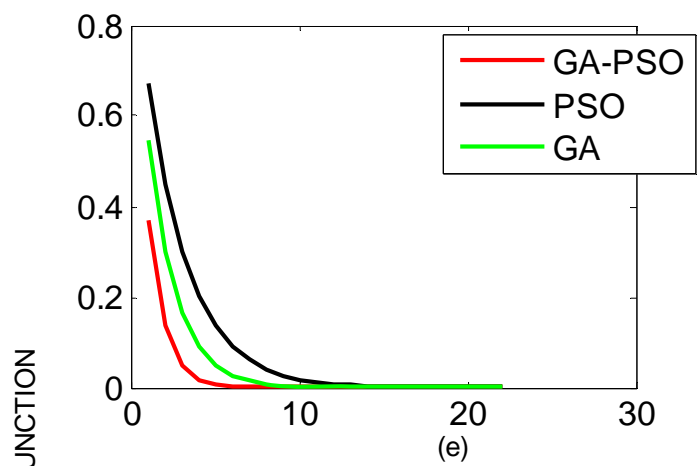

(e)

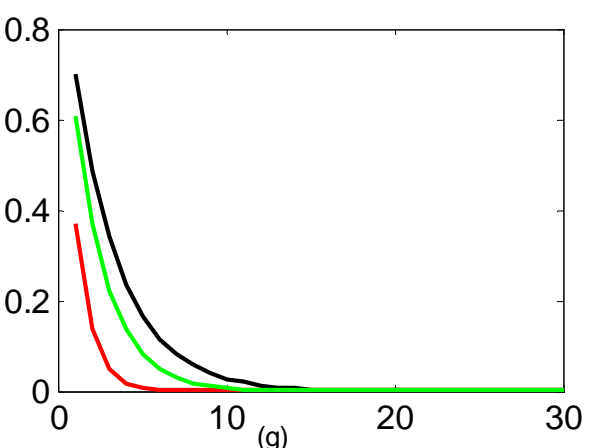

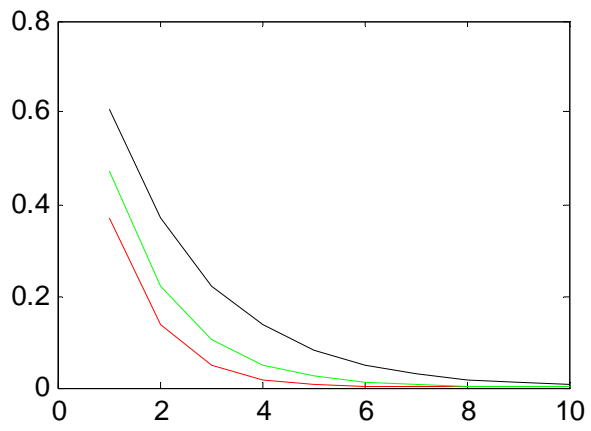

(b)

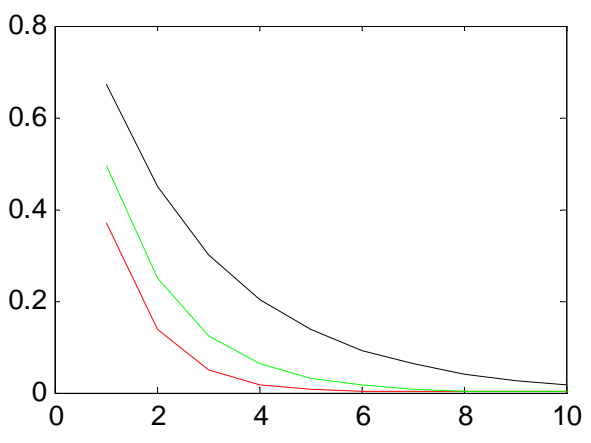

(d)
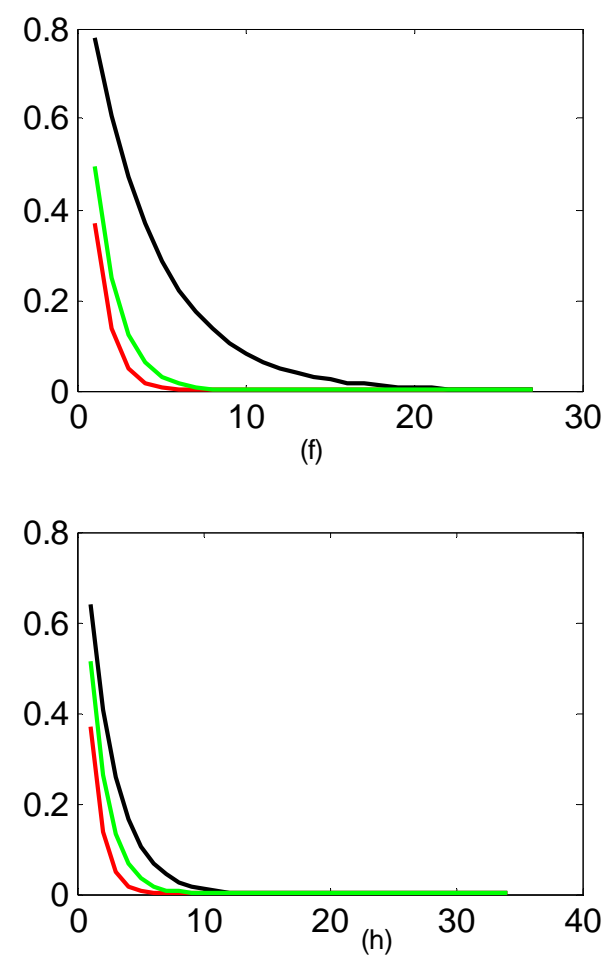

Fig 3. Convergence plot of the three methods to solve the benchmark problems 
TABLE III. Simulation results of the three methods to solve the benchmark problems

\begin{tabular}{|c|c|c|c|c|}
\hline Case & Method & Best & Mean & Standard Deviation \\
\hline \multirow[t]{3}{*}{$F_{162}$} & PSO & 0.0002 & 0.0004 & 0.0021 \\
\hline & GA & 0.0003 & 0.0003 & 0.0018 \\
\hline & Hybrid GA-PSO & 0.0000 & 0.0000 & 0.0007 \\
\hline \multirow[t]{3}{*}{$\mathrm{F}_{163}$} & PSO & 0.0006 & 0.0008 & 0.0022 \\
\hline & GA & 0.0006 & 0.0007 & 0.0017 \\
\hline & Hybrid GA-PSO & 0.0000 & 0.0000 & 0.0007 \\
\hline \multirow[t]{3}{*}{$\mathrm{F}_{164}$} & PSO & 21.43 & 21.45 & 0.0021 \\
\hline & GA & 21.38 & 21.39 & 0.0018 \\
\hline & Hybrid GA-PSO & 21.35 & 21.35 & 0.0006 \\
\hline \multirow[t]{3}{*}{$\mathrm{F}_{168}$} & PSO & 0.0000 & 0.0002 & 0.0024 \\
\hline & GA & 0.0000 & 0.0004 & 0.0019 \\
\hline & Hybrid GA-PSO & 0.0000 & 0.0000 & 0.0007 \\
\hline \multirow[t]{3}{*}{$\mathrm{F}_{174}$} & PSO & -0.0042 & -0.0044 & 0.0021 \\
\hline & GA & -0.0039 & -0.0041 & 0.0018 \\
\hline & Hybrid GA-PSO & -0.0037 & -0.0037 & 0.0007 \\
\hline \multirow[t]{3}{*}{$\mathrm{F}_{137}$} & PSO & 0.0005 & 0.0006 & 0.0022 \\
\hline & GA & 0.0005 & 0.0005 & 0.0017 \\
\hline & Hybrid GA-PSO & 0.0000 & 0.0000 & 0.0007 \\
\hline \multirow[t]{3}{*}{$\mathrm{F}_{141}$} & PSO & 0.0003 & 0.0006 & 0.0021 \\
\hline & GA & 0.0003 & 0.0005 & 0.0018 \\
\hline & Hybrid GA-PSO & 0.0000 & 0.0000 & 0.0006 \\
\hline \multirow[t]{3}{*}{$\mathrm{F}_{144}$} & PSO & -78.48 & -78.51 & 0.0024 \\
\hline & GA & -78.45 & -78.47 & 0.0019 \\
\hline & Hybrid GA-PSO & -78.33 & -78.33 & 0.0007 \\
\hline \multirow[t]{3}{*}{$\mathrm{F}_{145}$} & PSO & 0.0004 & 0.0007 & 0.0022 \\
\hline & GA & 0.0004 & 0.0005 & 0.0019 \\
\hline & Hybrid GA-PSO & 0.0000 & 0.0000 & 0.0005 \\
\hline \multirow[t]{3}{*}{$\mathrm{F}_{175}$} & PSO & -0.3526 & -0.3529 & 0.0024 \\
\hline & GA & -0.3528 & -0.3531 & 0.0018 \\
\hline & Hybrid GA-PSO & -0.3523 & -0.3523 & 0.0007 \\
\hline
\end{tabular}

\section{CONCLUSION}

This research proposes a hybrid algorithm that integrates two well established methods, one the genetic algorithm (GA) and the other the particle swarm optimization (PSO) algorithm. Here the GA will be the main optimizer and the PSO will be used to guide the GA to locate optimal solutions quickly and effectively. The exploration capability of the PSO is utilized by the GA for both exploration and exhaustive search. Thus complex search spaces are well searched and quality solutions are swiftly determined. Several benchmark test problems are solved and the applicability of the proposed hybrid algorithm is well established. Based on the simulation results, the proposed algorithm can be applied for solving real time optimization problems 


\section{REFERENCES}

[1] Juang, C. F.. A hybrid of genetic algorithm and particle swarm optimization for recurrent network design. IEEE Transactions on Systems, Man, and Cybernetics, Part B (Cybernetics), 34(2), 997-1006, 2004

[2] Kao, Y. T., \& Zahara, E. A hybrid genetic algorithm and particle swarm optimization for multimodal functions. Applied Soft Computing, 8(2), 849-857, 2008

[3] Marinakis, Y., \& Marinaki, M.. A hybrid genetic-Particle Swarm Optimization Algorithm for the vehicle routing problem. Expert Systems with Applications, 37(2), 1446-1455, 2010.

[4] Kuo, R. J., \& Han, Y. S.. A hybrid of genetic algorithm and particle swarm optimization for solving bi-level linear programming problem-A case study on supply chain model. Applied Mathematical Modelling, 35(8), 3905-3917, 2011.

[5] Li, S., Wu, X., \& Tan, M. Gene selection using hybrid particle swarm optimization and genetic algorithm. Soft Computing, 12(11), 1039-1048, 2008

[6] Shi, X. H., Liang, Y. C., Lee, H. P., Lu, C., \& Wang, L. M. An improved GA and a novel PSO-GA-based hybrid algorithm. Information Processing Letters, 93(5), 255-261, 2005.

[7] Duan, H., Luo, Q., Shi, Y., \& Ma, G. Hybrid particle swarm optimization and genetic algorithm for multi-uav formation reconfiguration. IEEE Computational Intelligence Magazine, 8(3), 16-27, 2013.

[8] Kuo, R. J., \& Lin, L. M.. Application of a hybrid of genetic algorithm and particle swarm optimization algorithm for order clustering. Decision Support Systems, 49(4), 451-462, 2010.

[9] Moradi, M. H., \& Abedini, M. A combination of genetic algorithm and particle swarm optimization for optimal DG location and sizing in distribution systems. International Journal of Electrical Power \& Energy Systems, 34(1), 66-74, 2012.

[10] Asokan, P., Jerald, J., Arunachalam, S., \& Page, T. Application of adaptive genetic algorithm and particle swarm optimisation in scheduling of jobs and AS/RS in FMS. International Journal of Manufacturing Research, 3(4), 393-405, 2008

[11] Holland, J. H., Genetic algorithms. Scientific American, 267(1), 66-72, 1992.

[12] Shi, Y., \& Eberhart, R. A modified particle swarm optimizer. In Evolutionary Computation Proceedings, 1998. IEEE World Congress on Computational Intelligence., The 1998 IEEE International Conference on (pp. 69-73). IEEE, 1998.

[13] Eberhart, R. C., \& Shi, Y., Comparison between genetic algorithms and particle swarm optimization. In International Conference on Evolutionary Programming (pp. 611-616), 1998.

[14] Jamil, M., \& Yang, X. S., A literature survey of benchmark functions for global optimisation problems. International Journal of Mathematical Modelling and Numerical Optimisation, 4(2), 150-194, 2013.

[15] Grosan, C., \& Abraham, A., Hybrid evolutionary algorithms: methodologies, architectures, and reviews. In Hybrid evolutionary algorithms (pp. 1-17), 2007.

\section{AUTHOR PROFILE}

V. Jagan Mohan, is a research scholar in the Department of Electrical \& Electronics Engineering, Karpagam Academy of Higher Education, Coimbatore, he is doing his research in the field of soft computing application in power system optimization problems.

T. Arul Dass Albert, is a professor and research supervisor in the Department of Electrical \& Electronics Engineering, Karpagam Academy of Higher Education, Coimbatore, he is doing his research in the field of soft computing application in power system optimization problems. 